\title{
NAKAYAMA AUTOMORPHISMS OF FROBENIUS CELLULAR ALGEBRAS
}

\author{
YANBO LI
}

(Received 7 January 2012; accepted 6 May 2012)

\begin{abstract}
Let $A$ be a finite-dimensional Frobenius cellular algebra with cell datum $(\Lambda, M, C, i)$. Take a nondegenerate bilinear form $f$ on $A$. In this paper, we study the relationship among $i, f$ and a certain Nakayama automorphism $\alpha$. In particular, we prove that the matrix associated with $\alpha$ with respect to the cellular basis is uni-triangular under a certain condition.
\end{abstract}

2010 Mathematics subject classification: primary 16W10, 16W20; secondary 16D50.

Keywords and phrases: Frobenius algebras, cellular algebras, Nakayama automorphisms.

\section{Introduction and preliminaries}

Cellular algebras were introduced by Graham and Lehrer [2] in 1996. The classical examples of cellular algebras include Hecke algebras of finite type [1], Ariki-Koike algebras, Brauer algebras [2], partition algebras [7], and Birman-Wenzl algebras [8].

It is well known that each symmetric algebra is Frobenius. However, a Frobenius algebra need not be symmetric, even in the cellular case. We refer the reader to [5] for related counterexamples. It is natural to consider how far a Frobenius cellular algebra is from being symmetric. A Frobenius algebra $A$ is symmetric if and only if a certain Nakayama automorphism is an identical mapping. This motivates us to study the socalled Nakayama automorphism. In this paper, we will describe the form of a certain Nakayama automorphism in the cellular background.

More precisely, the main results of this note are as follows. If $A$ is a finitedimensional Frobenius cellular algebra with cell datum $(\Lambda, M, C, i)$ and $f$ is a nondegenerate bilinear form on $A$, then the fact that one of the following three statements holds implies that the others are equivalent: (1) both the left and right dual bases of the cellular basis are cellular; (2) $A$ is symmetric; (3) $f(a, 1)=f(i(a), 1)$ for all $a \in A$. In particular, we prove that the matrix associated with a certain Nakayama automorphism with respect to the cellular basis is uni-triangular when (1) holds.

Let us state some basic facts concerning Frobenius algebras which will be used in later proofs. Let $K$ be a field and let $A$ be a finite-dimensional $K$-algebra. Suppose

(c) 2012 Australian Mathematical Publishing Association Inc. 0004-9727/2012 \$16.00 
that there exists a $K$-bilinear form $f: A \times A \rightarrow K$. We say that $f$ is nondegenerate if the determinant of the matrix $\left(f\left(a_{i}, a_{j}\right)\right)_{a_{i}, a_{j} \in B}$ is not zero for some basis $B$ of $A$. We call $f$ associative if $f(a b, c)=f(a, b c)$ for all $a, b, c \in A$.

Definition 1.1. A $K$-algebra $A$ is called Frobenius if there is a nondegenerate associative bilinear form $f$ on $A$. We call $A$ a symmetric algebra if, in addition, $f$ is symmetric, that is, $f(a, b)=f(b, a)$ for all $a, b \in A$.

For Frobenius algebras, Holm and Zimmermann proved the following lemma.

Lemma 1.2 [4, Lemma 2.7]. Let A be a finite-dimensional Frobenius algebra. Then an automorphism $\alpha$ of $A$ is a Nakayama automorphism if and only if

$$
f(a, b)=f(\alpha(b), a)
$$

Let $A$ be a Frobenius algebra with a basis $B=\left\{a_{i} \mid i=1, \ldots, n\right\}$. Let us take a nondegenerate associative bilinear form $f$. Define a $K$-linear map $\tau: A \rightarrow K$ by

$$
\tau(a)=f(a, 1)
$$

We call $d=\left\{d_{i} \mid i=1, \ldots, n\right\}$ the right dual basis of $B$; it is uniquely determined by the requirement that $\tau\left(a_{i} d_{j}\right)=\delta_{i j}$ for all $i, j=1, \ldots, n$. Similarly, the left dual basis $D=\left\{D_{i} \mid i=i, \ldots, n\right\}$ is determined by the requirement that $\tau\left(D_{j} a_{i}\right)=\delta_{i j}$. Define a $K$-linear map $\alpha: A \rightarrow A$ by

$$
\alpha\left(d_{i}\right)=D_{i}
$$

It follows from Lemma 1.2 that $\alpha$ is a Nakayama automorphism of $A$. If $A$ is a symmetric algebra, then $\alpha$ is the identity map and the right dual basis coincides with the left dual basis.

For any $i, j, k \in 1,2, \ldots, n$, let us write

$$
a_{i} a_{j}=\sum_{k} r_{i j k} a_{k},
$$

where $r_{i j k} \in K$. Fixing a $\tau$ for $A$, we have the following lemma about structure constants $r_{i j k}$.

Lemma 1.3. Let A be a Frobenius algebra with a basis $B$ and dual bases $d$ and $D$. Then the following hold.

(1) $a_{i} d_{j}=\sum_{k} r_{k i j} d_{k}$.

(2) $D_{i} a_{j}=\sum_{k} r_{j k i} D_{k}$.

Proof. (1) Suppose that $a_{i} d_{j}=\sum_{k} r_{k} d_{k}$. Left multiplying by $a_{k_{0}}$ on both sides of the equation and applying $\tau$, where $k_{0} \in 1, \ldots, n$, we get $\tau\left(a_{k_{0}} a_{i} d_{j}\right)=r_{k_{0}}$. Then $r_{k_{0}}=r_{k_{0}, i, j}$. (2) is proved similarly. 


\section{Frobenius cellular algebras}

First, let us recall the definition of a cellular algebra given in [2] by Graham and Lehrer.

Definition 2.1 [2]. Let $R$ be a commutative ring with identity. An associative unital $R$-algebra is called a cellular algebra with cell datum $(\Lambda, M, C, i)$ if the following conditions are satisfied.

(C1) The finite set $\Lambda$ is a poset. Associated with each $\lambda \in \Lambda$, there is a finite set $M(\lambda)$. The algebra $A$ has an $R$-basis $\left\{C_{S, T}^{\lambda} \mid S, T \in M(\lambda), \lambda \in \Lambda\right\}$.

(C2) The map $i$ is an $R$-linear anti-automorphism of $A$ with $i^{2}=i d$ which sends $C_{S, T}^{\lambda}$ to $C_{T, S}^{\lambda}$.

(C3) If $\lambda \in \Lambda$ and $S, T \in M(\lambda)$, then for any element $a \in A$,

$$
a C_{S, T}^{\lambda} \equiv \sum_{S^{\prime} \in M(\lambda)} r_{a}\left(S^{\prime}, S\right) C_{S^{\prime}, T}^{\lambda} \quad(\bmod A(<\lambda))
$$

where $r_{a}\left(S^{\prime}, S\right) \in R$ is independent of $T$ and where $A(<\lambda)$ is the $R$-submodule of $A$ generated by $\left\{C_{S^{\prime \prime}, T^{\prime \prime}}^{\mu} \mid \mu<\lambda, S^{\prime \prime}, T^{\prime \prime} \in M(\mu)\right\}$.

If we apply $i$ to the equation in (C3),

$$
C_{T, S}^{\lambda} i(a) \equiv \sum_{S^{\prime} \in M(\lambda)} r_{a}\left(S^{\prime}, S\right) C_{T, S^{\prime}}^{\lambda} \quad(\bmod A(<\lambda)) .
$$

Let $A$ be a finite-dimensional Frobenius cellular $K$-algebra with cell datum $(\Lambda, M, C, i)$ and a nondegenerate associative bilinear form $f$. Denote the right dual basis by $d=\left\{d_{S, T}^{\lambda} \mid S, T \in M(\lambda), \lambda \in \Lambda\right\}$, which satisfies

$$
\tau\left(C_{S, T}^{\lambda} d_{U, V}^{\mu}\right)=\delta_{\lambda \mu} \delta_{S, V} \delta_{T, U}
$$

Denote the left dual basis by $D=\left\{D_{S, T}^{\lambda} \mid S, T \in M(\lambda), \lambda \in \Lambda\right\}$, which satisfies

$$
\tau\left(D_{U, V}^{\mu} C_{S, T}^{\lambda}\right)=\delta_{\lambda \mu} \delta_{S, V} \delta_{T, U}
$$

For $\mu \in \Lambda$, let $A_{d}(>\mu)$ be the $R$-submodule of $A$ generated by

$$
\left\{d_{P, Q}^{\eta} \mid P, Q \in M(\eta), \mu<\eta\right\},
$$

and let $A_{D}(>\mu)$ be the $R$-submodule of $A$ generated by

$$
\left\{D_{P, Q}^{\eta} \mid P, Q \in M(\eta), \mu<\eta\right\} .
$$

For $\lambda, \mu \in \Lambda, S, T \in M(\lambda), U, V \in M(\mu)$, write

$$
C_{S, T}^{\lambda} C_{U, V}^{\mu}=\sum_{\epsilon \in \Lambda, X, Y \in M(\epsilon)} r_{(S, T, \lambda),(U, V, \mu),(X, Y, \epsilon)} C_{X, Y}^{\epsilon} .
$$

The following lemma is a corollary of Lemma 1.3 and Definition 2.1. It plays an important role in this note. 
LEMMA 2.2. Let $A$ be a finite-dimensional Frobenius cellular algebra with cell datum $(\Lambda, M, C, i)$. Let $d$ be the right dual basis and $D$ the left dual basis determined by $a$ given $\tau$. Then for arbitrary $\lambda, \mu \in \Lambda$ and $S, T, P, Q \in M(\lambda), U, V \in M(\mu)$, the following equations hold.

$$
\begin{gathered}
D_{U, V}^{\mu} C_{S, T}^{\lambda}=\sum_{\epsilon \in \Lambda, X, Y \in M(\epsilon)} r_{(S, T, \lambda),(Y, X \epsilon),(V, U, \mu)} D_{X, Y}^{\epsilon} \cdot \\
C_{S, T}^{\lambda} d_{U, V}^{\mu}=\sum_{\epsilon \in \Lambda, X, Y \in M(\epsilon)} r_{(Y, X, \epsilon),(S, T, \lambda),(V, U, \mu)} d_{X, Y}^{\epsilon} \cdot \\
C_{S, T}^{\lambda} d_{P, Q}^{\lambda}=0 \quad \text { if } T \neq P . \\
D_{P, Q}^{\lambda} C_{S, T}^{\lambda}=0 \quad \text { if } Q \neq S . \\
C_{S, T}^{\lambda} d_{U, V}^{\mu}=0 \quad \text { if } \mu \not \leq \lambda . \\
D_{U, V}^{\mu} C_{S, T}^{\lambda}=0 \quad \text { if } \mu \not \leq \lambda .
\end{gathered}
$$

Proof. Clearly, (1), (2) are corollaries of Lemma 1.3; (3)-(6) are corollaries of (1) and (2).

Now we are ready to study dual bases of the cellular basis of a Frobenius cellular algebra.

Lemma 2.3. Let $A$ be a Frobenius cellular algebra. For $\mu \in \Lambda$ and $U, V \in M(\mu)$ and an element $a \in A$,

$$
\begin{aligned}
& a d_{U, V}^{\mu} \equiv \sum_{U^{\prime} \in M(\mu)} r_{i(a)}\left(U, U^{\prime}\right) d_{U^{\prime}, V}^{\mu} \quad\left(\bmod A_{d}(>\mu)\right) . \\
& d_{U, V}^{\mu} a \equiv \sum_{V^{\prime} \in M(\mu)} r_{a}\left(V, V^{\prime}\right) d_{U, V^{\prime}}^{\mu} \quad\left(\bmod A_{d}(>\mu)\right) . \\
& a D_{U, V}^{\mu} \equiv \sum_{U^{\prime} \in M(\mu)} r_{i(a)}\left(U, U^{\prime}\right) D_{U^{\prime}, V}^{\mu} \quad\left(\bmod A_{D}(>\mu)\right) . \\
& D_{U, V}^{\mu} a \equiv \sum_{V^{\prime} \in M(\mu)} r_{a}\left(V, V^{\prime}\right) D_{U, V^{\prime}}^{\mu} \quad\left(\bmod A_{D}(>\mu)\right) .
\end{aligned}
$$

Proof. (1) For arbitrary $C_{S, T}^{\lambda}$, it follows from (2) of Lemma 2.2 that

$$
C_{S, T}^{\lambda} d_{U, V}^{\mu}=\sum_{\epsilon \in \Lambda, X, Y \in M(\epsilon)} r_{(Y, X, \epsilon),(S, T, \lambda),(V, U, \mu)} d_{X, Y}^{\epsilon} .
$$

By (C3) of Definition 2.1, if $\epsilon<\mu$, then $r_{(Y, X, \epsilon),(S, T, \lambda),(V, U, \mu)}=0$. Therefore,

$$
C_{S, T}^{\lambda} d_{U, V}^{\mu} \equiv \sum_{X, Y \in M(\mu)} r_{(Y, X, \mu),(S, T, \lambda),(V, U, \mu)} d_{X, Y}^{\mu} \quad\left(\bmod A_{d}(>\mu)\right) .
$$

By $\left(\mathrm{C}^{\prime}\right)$ of Definition 2.1, if $Y \neq V$, then $r_{(Y, X, \mu),(S, T, \lambda),(V, U, \mu)}=0$. So

$$
C_{S, T}^{\lambda} d_{U, V}^{\mu} \equiv \sum_{X \in M(\mu)} r_{(V, X, \mu),(S, T, \lambda),(V, U, \mu)} d_{X, V}^{\mu} \quad\left(\bmod A_{D}(>\mu)\right) .
$$


Clearly, for arbitrary $X \in M(\mu)$,

$$
r_{(V, X, \mu),(S, T, \lambda),(V, U, \mu)}=r_{C_{T, S}^{\lambda}}(U, X),
$$

which is independent of $V$. Since $C_{S, T}^{\lambda}$ is arbitrary,

$$
a d_{U, V}^{\mu} \equiv \sum_{U^{\prime} \in M(\mu)} r_{i(a)}\left(U, U^{\prime}\right) d_{U^{\prime}, V}^{\mu} \quad\left(\bmod A_{d}(>\mu)\right)
$$

for all $a \in A$. By Definition 2.1, $r_{i(a)}\left(U, U^{\prime}\right)$ is independent of $V$.

(4) is proved similarly.

Applying $\alpha$ on both sides of (1), we get (3). Similarly, applying $\alpha^{-1}$ on both sides of (4), we get (2).

Lemma 2.3 implies that dual bases of the cellular basis satisfy Definition 2.1 (C3) with respect to the opposite order on $\Lambda$. However, (C2) does not hold, that is, either $i\left(d_{S, T}^{\lambda}\right)=d_{T, S}^{\lambda}$ or $i\left(D_{S, T}^{\lambda}\right)=D_{T, S}^{\lambda}$ need not be true in general. We give an example which was constructed by Koenig and $\mathrm{Xi}$ in [5].

Example 2.4. Let $K$ be a field. Let us take $\lambda \in K$ with $\lambda \neq 0$ and $\lambda \neq 1$. Let

$$
A=K\langle a, b, c, d\rangle / I,
$$

where $I$ is generated by

$$
a^{2}, b^{2}, c^{2}, d^{2}, a b, a c, b a, b d, c a, c d, d b, d c, c b-\lambda b c, a d-b c, d a-b c .
$$

If we define $\tau$ by $\tau(1)=\tau(a)=\tau(b)=\tau(c)=\tau(d)=0$ and $\tau(b c)=1$ and define an involution $i$ on $A$ to be fixing $a$ and $d$, but interchanging $b$ and $c$, then $A$ is a Frobenius cellular algebra with a cellular basis

$$
b c ; \quad \begin{array}{lll}
a & b \\
& c & d
\end{array} ; 1
$$

The right dual basis is

$$
1 ; \quad \begin{array}{cc}
d & c \\
b / \lambda & a
\end{array} ; \quad b c
$$

The left dual basis is

$$
1 ; \quad \begin{array}{cc}
d & c / \lambda \\
b & a
\end{array} ; \quad b c
$$

Clearly, $i(c)=b \neq b / \lambda, i(b)=c \neq c / \lambda$.

Example 2.4 implies that for a Frobenius cellular algebra, the dual bases of a cellular basis need not be cellular again. The following result reveals a relation among $\alpha, i$ and $\tau$.

Theorem 2.5. Let $A$ be a Frobenius cellular algebra with cell datum $(\Lambda, M, C, i)$. If one of the following three statements holds, then the other two are equivalent. 
(1) $i\left(d_{S, T}^{\lambda}\right)=d_{T, S}^{\lambda}$ and $i\left(D_{S, T}^{\lambda}\right)=D_{T, S}^{\lambda}$ for arbitrary $\lambda \in \Lambda$ and $S, T \in M(\lambda)$.

(2) $\alpha=$ id, that is, $A$ is a symmetric algebra.

(3) $\tau(a)=\tau(i(a))$ for all $a \in A$.

Proof. It is enough to prove that if two of the statements hold then the other one is true.

Suppose that (1) and (2) hold. Since $A$ is symmetric, the right dual basis is equal to the left dual basis. Denote the dual basis by $\left\{D_{S, T}^{\lambda} \mid \lambda \in \Lambda, S, T \in M(\lambda)\right\}$. Let

$$
1=\sum_{\varepsilon \in \Lambda, X, Y \in M(\varepsilon)} r_{X, Y, \varepsilon} D_{X, Y}^{\varepsilon}
$$

Then

$$
\tau\left(C_{S, T}^{\lambda}\right)=\tau\left(C_{S, T}^{\lambda} \sum_{\varepsilon \in \Lambda, X, Y \in M(\varepsilon)} r_{X, Y, \varepsilon} D_{X, Y}^{\varepsilon}\right)=r_{T, S, \lambda} .
$$

On the other hand, it follows from (1) that

$$
1=i(1)=\sum_{\varepsilon \in \Lambda, X, Y \in M(\varepsilon)} r_{X, Y, \varepsilon} i\left(D_{X, Y}^{\varepsilon}\right)=\sum_{\varepsilon \in \Lambda, X, Y \in M(\varepsilon)} r_{X, Y, \varepsilon} D_{Y, X}^{\varepsilon} .
$$

Then

$$
\tau\left(C_{T, S}^{\lambda}\right)=\tau\left(\sum_{\varepsilon \in \Lambda, X, Y \in M(\varepsilon)} r_{X, Y, \varepsilon} D_{Y, X}^{\varepsilon} C_{T, S}^{\lambda}\right)=r_{T, S, \lambda} .
$$

Now we obtain $\tau\left(C_{S, T}^{\lambda}\right)=\tau\left(C_{T, S}^{\lambda}\right)$ for any $\lambda \in \Lambda$ and $S, T \in M(\lambda)$. Hence $\tau(a)=\tau(i(a))$ for all $a \in A$.

Assume that (1) and (3) hold. Then

$$
\tau\left(d_{U, V}^{\mu} C_{T, S}^{\lambda}\right)=\tau\left(C_{S, T}^{\lambda} d_{V, U}^{\mu}\right)=\delta_{\lambda \mu} \delta_{T V} \delta_{S U} .
$$

This implies that $d_{U, V}^{\mu}=D_{U, V}^{\mu}$ for any $\mu \in \Lambda$ and $U, V \in M(\mu)$ by the definition of dual bases. Hence the algebra $A$ is symmetric.

Assume that (2) and (3) hold. Since $A$ is symmetric, we can denote the dual basis by $\left\{D_{S, T}^{\lambda} \mid \lambda \in \Lambda, S, T \in M(\lambda)\right\}$. It follows from (3) and $\tau\left(C_{S, T}^{\lambda} D_{T, S}^{\lambda}\right)=1$ that

$$
\tau\left(i\left(D_{T, S}^{\lambda}\right) C_{T, S}^{\lambda}\right)=1 \text {. }
$$

On the other hand, $\tau\left(C_{U, V}^{\mu} D_{T, S}^{\lambda}\right)=0$ if $(U, V, \mu) \neq(S, T, \lambda)$. This implies that

$$
\tau\left(i\left(D_{T, S}^{\lambda}\right) C_{V, U}^{\lambda}\right)=0
$$

if $(U, V, \mu) \neq(S, T, \lambda)$. Note that the dual basis is uniquely determined by $\tau$. Then

$$
i\left(D_{S, T}^{\lambda}\right)=D_{T, S}^{\lambda} \cdot
$$

The proof is complete.

Remark 2.6. Graham in [3] showed that for a symmetric cellular algebra $A$ with cell datum $(\Lambda, M, C, i)$, the dual basis is again cellular with respect to the opposite order on $\Lambda$ if $\tau(a)=\tau(i(a))$ for all $a \in A$. 


\section{Nakayama automorphisms of Frobenius cellular algebras}

The cellularity of dual bases and the symmetry of the algebra are connected by Theorem 2.5. In this section, we describe this relation by investigating the Nakayama automorphism $\alpha$ defined by $\alpha\left(d_{S, T}^{\lambda}\right)=D_{S, T}^{\lambda}$. For convenience, we extend the poset $\Lambda$ to a totally ordered set. Then the main result of this section is as follows.

Theorem 3.1. Let A be a finite-dimensional Frobenius cellular $K$-algebra with a cell datum $(\Lambda, M, C, i)$. Suppose that both the dual bases are cellular with respect to the opposite order on $\Lambda$. Then

$$
\alpha\left(C_{S, T}^{\lambda}\right) \equiv C_{S, T}^{\lambda} \quad(\bmod A(<\lambda)) .
$$

Proof. Suppose that

$$
\alpha\left(C_{S, T}^{\lambda}\right)=\sum_{\varepsilon \in \Lambda, X, Y \in M(\varepsilon)} r_{X, Y, \varepsilon} C_{X, Y}^{\varepsilon} .
$$

Let us first prove some claims.

Claim 1. Let $\varepsilon>\lambda$ and $X, Y \in M(\varepsilon)$. Then $r_{X, Y, \varepsilon}=0$.

Suppose that there exist $\mu>\lambda$ and $U, V \in M(\lambda)$ such that $r_{U, V, \mu} \neq 0$. Without loss of generality, assume that $\mu \in \Lambda$ is the maximal element satisfying this condition, that is, if $\mu<\varepsilon$, then $r_{X, Y, \varepsilon}=0$ for all $X, Y \in M(\varepsilon)$. Then, from Lemma 2.2,

$$
\alpha\left(C_{S, T}^{\lambda}\right) D_{V, U}^{\mu}=\alpha\left(C_{S, T}^{\lambda} d_{V, U}^{\mu}\right)=0 .
$$

On the other hand, it follows from $i\left(D_{V, U}^{\mu}\right)=D_{U, V}^{\mu}$ that

$$
\tau\left(i\left(\alpha\left(C_{S, T}^{\lambda}\right) D_{V, U}^{\mu}\right)\right)=\tau\left(r_{U, V, \mu} D_{U, V}^{\mu} C_{V, U}^{\mu}\right)=r_{U, V, \mu} \neq 0 .
$$

This is a contradiction and the claim is proved.

This claim implies that $\alpha(A(<\lambda))=A(<\lambda)$. But the form

$$
\alpha^{-1}(A(<\lambda))=A(<\lambda)
$$

is more useful to us.

Claim 2. $r_{P, Q, \lambda}=0$ if $Q \neq T$.

Suppose that $r_{P, Q, \lambda} \neq 0$ and $Q \neq T$. Then, by Lemma 2.2, $Q \neq T$ implies that

$$
\alpha\left(C_{S, T}^{\lambda}\right) D_{Q, P}^{\lambda}=\alpha\left(C_{S, T}^{\lambda} d_{Q, P}^{\lambda}\right)=0 .
$$

However, still by Lemma 2.2,

$$
\begin{aligned}
\tau\left(i\left(\alpha\left(C_{S, T}^{\lambda}\right) D_{Q, P}^{\lambda}\right)\right) & =\tau\left(D_{P, Q}^{\lambda} i\left(\alpha\left(C_{S, T}^{\lambda}\right)\right)\right) \\
& =\tau\left(D_{P, Q}^{\lambda} \sum_{X, Y \in M(\lambda)} r_{X, Y, \lambda} C_{Y, X}^{\lambda}\right) \\
& =\tau\left(\sum_{X, Y \in M(\lambda)} r_{X, Y, \lambda} D_{P, Q}^{\lambda} C_{Y, X}^{\lambda}\right)=r_{P, Q, \lambda} \neq 0 .
\end{aligned}
$$

Hence $i\left(\alpha\left(C_{S, T}^{\lambda}\right) D_{Q, P}^{\lambda}\right) \neq 0$, or $\alpha\left(C_{S, T}^{\lambda}\right) D_{Q, P}^{\lambda} \neq 0$. This is a contradiction. 
Claim 3. $r_{X, T, \lambda}=0$ if $X \neq S$.

By Claims 1 and 2,

$$
\alpha\left(C_{S, T}^{\lambda}\right) \equiv \sum_{X \in M(\lambda)} r_{X, T, \lambda} C_{X, T}^{\lambda} \quad(\bmod (A<\lambda)) .
$$

Then it follows from $\alpha^{-1}(A(<\lambda))=A(<\lambda)$ that

$$
C_{S, T}^{\lambda} \equiv \sum_{X \in M(\lambda)} r_{X, T, \lambda} \alpha^{-1}\left(C_{X, T}^{\lambda}\right) \quad(\bmod (A<\lambda))
$$

Left multiplying by $d_{T, V}^{\lambda}$ on both sides, we have from Lemma 2.2 that

$$
\begin{aligned}
d_{T, V}^{\lambda} C_{S, T}^{\lambda} & =\sum_{X \in M(\lambda)} r_{X, T, \lambda} d_{T, V}^{\lambda} \alpha^{-1}\left(C_{X, T}^{\lambda}\right) \\
& =\sum_{X \in M(\lambda)} r_{X, T, \lambda} \alpha^{-1}\left(D_{T, V}^{\lambda} C_{X, T}^{\lambda}\right) \\
& =r_{V, T, \lambda} \alpha^{-1}\left(D_{T, V}^{\lambda} C_{V, T}^{\lambda}\right) .
\end{aligned}
$$

On the other hand, by Lemma 2.2, $i\left(d_{T, V}^{\lambda} C_{S, T}^{\lambda}\right)=C_{T, S}^{\lambda} d_{V, T}^{\lambda}=0$ if $V \neq S$. Thus $d_{T, V}^{\lambda} C_{S, T}^{\lambda}=0$ if $V \neq S$. Moreover, the facts that $D_{T, V}^{\lambda} C_{V, T}^{\lambda} \neq 0$ and $\alpha$ is an automorphism of $A$ imply that $\alpha^{-1}\left(D_{T, V}^{\lambda} C_{V, T}^{\lambda}\right) \neq 0$. Now we get $r_{V, T \lambda}=0$ if $V \neq S$. Then the claim is proved.

We have proved that the Nakayama automorphism $\alpha$ is of the form

$$
\alpha\left(C_{S, T}^{\lambda}\right) \equiv r_{S, T, \lambda} C_{S, T}^{\lambda} \quad(\bmod (A<\lambda)) .
$$

It is sufficient to show that $r_{S, T, \lambda}=1$. We claim that $C_{P, Q}^{\eta} D_{T, S}^{\lambda}=0$ if $\eta<\lambda$. In fact, if $C_{P, Q}^{\eta} D_{T, S}^{\lambda} \neq 0$, then $i\left(C_{P, Q}^{\eta} D_{T, S}^{\lambda}\right) \neq 0$, that is, $D_{S, T}^{\lambda} C_{Q, P}^{\eta} \neq 0$. But Lemma 2.2 tells us that this is impossible. Now it follows from this claim and Lemma 2.2 that

$$
\begin{aligned}
r_{S, T, \lambda} C_{S, T}^{\lambda} D_{T, S}^{\lambda} & =\alpha\left(C_{S, T}^{\lambda}\right) D_{T, S}^{\lambda} \\
& =\alpha\left(C_{S, T}^{\lambda} d_{T, S}^{\lambda}\right) \\
& =\alpha\left(\sum_{\varepsilon \in \Lambda, X, Y \in M(\varepsilon)} r_{(Y, X, \varepsilon),(S, T, \lambda),(S, T, \lambda)} d_{X, Y}^{\varepsilon}\right) \\
& =\sum_{\varepsilon \in \Lambda, X, Y \in M(\varepsilon)} r_{(Y, X, \varepsilon),(S, T, \lambda),(S, T, \lambda)} D_{X, Y}^{\varepsilon} .
\end{aligned}
$$

On the other hand,

$$
\begin{aligned}
r_{S, T, \lambda} C_{S, T}^{\lambda} D_{T, S}^{\lambda} & =r_{S, T, \lambda} i\left(D_{S, T}^{\lambda} C_{T, S}^{\lambda}\right) \\
& =i\left(\sum_{\varepsilon \in \Lambda, X, Y \in M(\varepsilon)} r_{S, T, \lambda} r_{(T, S, \lambda),(Y, X, \varepsilon),(T, S, \lambda)} D_{X, Y}^{\varepsilon}\right)
\end{aligned}
$$




$$
\begin{aligned}
& =\sum_{\varepsilon \in \Lambda, X, Y \in M(\varepsilon)} r_{S, T, \lambda} r_{(T, S, \lambda),(Y, X, \varepsilon),(T, S, \lambda)} D_{Y, X}^{\varepsilon} \\
& =\sum_{\varepsilon \in \Lambda, X, Y \in M(\varepsilon)} r_{S, T, \lambda} r_{(T, S, \lambda),(X, Y, \varepsilon),(T, S, \lambda)} D_{X, Y}^{\varepsilon} .
\end{aligned}
$$

Note that $\alpha$ is an automorphism of $A$; then clearly $r_{S, T, \lambda} \neq 0$. Then it follows from $C_{S, T}^{\lambda} D_{T, S}^{\lambda} \neq 0$ that $r_{S, T, \lambda} C_{S, T}^{\lambda} D_{T, S}^{\lambda} \neq 0$. This implies that there exist $\mu \in \Lambda$ and $U, V \in M(\mu)$ such that $r_{(T, S, \lambda),(U, V, \mu),(T, S, \lambda)} \neq 0$ and

$$
r_{S, T, \lambda} r_{(T, S, \lambda),(U, V, \mu),(T, S, \lambda)}=r_{(V, U, \mu),(S, T, \lambda),(S, T, \lambda)} .
$$

From (C3)' of Definition 2.1,

$$
r_{(T, S, \lambda),(U, V, \mu),(T, S, \lambda)}=r_{(V, U, \mu),(S, T, \lambda),(S, T, \lambda)} .
$$

This implies that $r_{S, T, \lambda}=1$ and we complete the proof.

REMARK 3.2. In fact, the condition $i\left(d_{S, T}^{\lambda}\right)=d_{T, S}^{\lambda}$ could be weakened to

$$
i\left(d_{S, T}^{\lambda}\right) \equiv d_{T, S}^{\lambda} \quad\left(\bmod \left(A_{d}(>\lambda)\right)\right) .
$$

We omit the details here.

In [6], the author studied the centres of symmetric cellular algebras. We generalise Theorem 1.1 of [6] to the Frobenius case in the present paper.

Let $A$ be a Frobenius cellular algebra. The Higman ideal of $Z(A)$ is defined by

$$
H(A)=\left\{\sum_{\lambda \in \Lambda, S, T \in M(\lambda)} C_{S, T}^{\lambda} a D_{S, T}^{\lambda} \mid a \in A\right\} .
$$

For any $\lambda \in \Lambda$ and $T \in M(\lambda)$, set $e_{\lambda}=\sum_{S \in M(\lambda)} C_{S, T}^{\lambda} D_{T, S}^{\lambda}$ and

$$
L(A)=\left\{\sum_{\lambda \in \Lambda} r_{\lambda} e_{\lambda} \mid r_{\lambda} \in R\right\} .
$$

Then we have the following corollary.

Corollary 3.3. Let $A$ be a Frobenius cellular algebra with cellular datum $(\Lambda, M, C, i)$ and both the right and left dual bases are cellular. Then $L(A)$ is an ideal of $Z(A)$ containing the Higman ideal.

Proof. The proof is similar to that in [6].

\section{Acknowledgements}

The author express his thanks to $\mathrm{Dr}$ Wei $\mathrm{Hu}$ for his valuable comments. He also acknowledges support from Fundamental Research Funds for the Central Universities (N110423007). 


\section{References}

[1] M. Geck, 'Hecke algebras of finite type are cellular', Invent. Math. 169 (2007), 501-517.

[2] J. J. Graham and G. I. Lehrer, 'Cellular algebras', Invent. Math. 123 (1996), 1-34.

[3] J. J. Graham, Modular Representations of Hecke Algebras and Related Algebras, PhD Thesis, Sydney University, 1995.

[4] T. Holm and A. Zimmermann, 'Deformed preprojective algebras of type L: Külshammer spaces and derived equivalences', J. Algebra 346 (2011), 116-146.

[5] S. Koenig and C. C. Xi, 'A self-injective cellular algebra is weakly symmetric', J. Algebra 228 (2000), 51-59.

[6] Y. Li, 'Centres of symmetric cellular algebras', Bull. Aust. Math. Soc. 82 (2010), 511-522.

[7] C. C. Xi, 'Partition algebras are cellular', Compositio Math. 119 (1999), 99-109.

[8] C. C. Xi, 'On the quasi-heredity of Birman-Wenzl algebras', Adv. Math. 154 (2000), 280-298.

YANBO LI, School of Mathematics and Statistics,

Northeastern University at Qinhuangdao, Qinhuangdao 066004, PR China

e-mail: liyanbo707@163.com 